\title{
Appendix: A Multi-objective Architecture Search for Generative Adversarial Networks
}

\author{
Masayuki Kobayashi \\ Yokohama National University \\ Yokohama, Japan \\ kobayashi-masayuki-xc@ynu.jp
}

\author{
Tomoharu Nagao \\ Yokohama National University \\ Yokohama, Japan \\ nagao@ynu.ac.jp
}

\section{SEARCH SPACE}

Unlike conventional architecture search methods, our method searches for better network architectures together with their hyper-parameters. In this study, we defined the following search spaces for both network architectures and hyper-parameters and optimized them by evolutionary algorithm.

\subsection{Node Functions}

Referring to the modern GAN architectures, we select the following operations as the node functions to form networks:

(1) conv: $3 \times 3$ convolution

(2) conv_sum: summation with conv

(3) res: $3 \times 3$ residual block

The summation is an operation that performs element-wise summation of two feature maps. This operation allows our method to form shortcut connections or branching layers. In addition, the search space of the generator also uses the following subset of upsampling operations in the output node:

(1) conv_nearest: nearest neighbor interpolation with conv

(2) conv_bilinaer: bilinear interpolation with conv

(3) res_nearest: nearest neighbor interpolation res

(4) res_bilinear: bilinear interpolation with res

In the same way as the generator, the discriminator uses the following subset of downsampling operations in the output node:

(1) conv_nearest: nearest neighbor interpolation with conv

(2) conv_bilinaer: bilinear interpolation with conv

(3) res_nearest: nearest neighbor interpolation res

(4) res_bilinear: bilinear interpolation with res

To fix the number of channels, we add a convolution operation on top of the generator. The rectified linear unit (ReLU) [11] and spectral normalization [9] are employed in all convolution operations. In addition, class-conditional batch normalization [2] and projection [10] are employed in the generator and the discriminator to feed the label information in the supervised image generation tasks.

\subsection{Hyper-parameters}

Referring to modern GAN training, we consider six hyper-parameter: the discriminator's learning rate $\alpha_{\text {gen }}$, the Adam $\beta_{2}$ parameter for each network, the gradient penalty strength $\gamma$, orthogonal regularization strength $\zeta$, and the discriminator update steps per iteration $n_{\text {dis }}$, and we explore these values through

- $\alpha_{\text {gen }}:\left[10^{-5}, 5.0 \times 10^{-5}, 8.0 \times 10^{-5}, 10^{-4}\right]$

- $\beta_{2 \text { gen }}$ and $\beta_{2 \text { dis }}:[0.9,0.99,0.99]$

- $\gamma:\left[10^{-3}, 10^{-2}, 10^{-1}, 0.5,1,2,3,5,10\right]$

- $\zeta:\left[10^{-5}, 5.0 \times 10^{-5}, 10^{-4}, 5.0 \times 10^{-4}, 10^{-3}, 10^{-2}\right]$

- $n_{\text {dis }}: 1,2$

We herein note that the generator's learning rate is fixed to $2.0 \times 10^{-4}$ to follow the two-timescale update rule (TTUR) [5].

\section{IMPLEMENTATION DETAILS}

We initialized the weight by using orthogonal initialization [12], and we used the Adam optimizer [6] with a mini-batch size of 64. During the search phase, the initial number of parameters was set to $0.3 \mathrm{M}$ in both models and was doubled at $1 / 2$ of the total generations. We expanded the number of parameters by increasing the width (number of channels) in each layer. For acceleration, 5,000 randomly generated images were used for evaluation (i.e., computing the fitness) during the search phase.

After the search process was completed, the best pair of networks was retrained for a specific number of iterations. On CIFAR-10 and STL-10, each network was retrained for $100 \mathrm{~K}$ and $150 \mathrm{~K}$ iterations with the mini-batch size of 128 . The initial number of channels of the 
networks was increased to approximately 64 to ensure that the generator and discriminator had approximately $10 \mathrm{M}$ parameters. Instead of adjusting the learning rate of optimizer, we used an exponential moving average of the generator's parameters with a decay rate of 0.9999 . Other training settings were the same as those used during the search phase. For a fair evaluation, 50,000 images were randomly generated to compute the inception score and FID.

For the network representation, we used the standard CGP [7, 8] with rows $N_{\mathrm{r}}=2$, columns $N_{\mathrm{c}}=5$, and level-back parameter $L=3$. We stack 3 CGPs to form the generator and the discriminator, respectively. We chose the number of generations as $N_{\text {gen }}=15$, population size as $N_{\text {pop }}=14$, and offspring size as $N_{\text {off }}=14$. We used non-dominated sorting genetic algorithms (NSGA-II) [1] for our multi-objective optimization.

\section{NETWORK ARCHITECTURES AND HYPER-PARAMETERS OBTAINED BY OUR METHOD}

In this section, we provide the network architectures discovered on our image generation tasks. Figure 1 shows one of the best performing architectures together with their hyper-parameters obtained by our method in unsupervised and supervised image generation tasks on CIFAR-10. From our experiments, there were several observations to note:

- Unlike previous works $[3,4]$, there seems to be no preference for upsampling operations. Both the nearest neighbor and bilinear interpolations were selected in our discovered models.

- We found that each hyper-parameter has a certain threshold that affects the performance. For instance, orthogonal regularization strengths $\zeta$ were mainly selected from below $1 \times 10^{-4}$, and the gradient penalties $\gamma$ were mostly selected from above 1 .

- We found that discriminator update steps tend to be selected to be 2 per generator step.

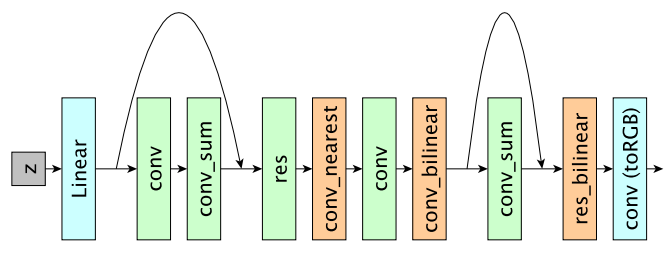

(a) Generator

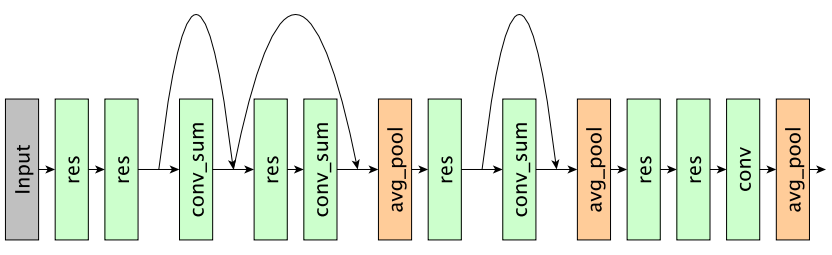

(b) Discriminator

Figure 1: One of the best performing architectures obtained by our method. Obtained hyper-parameters are $\alpha_{\text {gen }}=10^{-4}$, $\beta_{2_{\text {gen }}}=0.999, \beta_{2_{\text {dis }}}=0.999, \gamma=1.0, \zeta=10^{-5}, n_{\text {dis }}=2$.

\section{IMAGES GENERATED FROM OUR MODELS}

In this section, we provide some images generated from our models. The examples of generated images of CIFAR-10 and STL-10 are shown in Figure 2 and Figure 3, respectively. These images are not cherry-picked; they are 100 randomly sampled images from our latent space.

\section{REFERENCES}

[1] Kalyanmoy Deb, Amrit Pratap, Sameer Agarwal, and Tanaka Meyarivan. 2002. A fast and elitist multiobjective genetic algorithm: NSGA-II. IEEE Transactions on Evolutionary Computation 6, 2 (2002), 182-197.

[2] Vincent Dumoulin, Jonathon Shlens, and Manjunath Kudlur. 2017. A learned representation for artistic style. In ICLR.

[3] Chen Gao, Yunpeng Chen, Si Liu, Zhenxiong Tan, and Shuicheng Yan. 2019. AdversarialNAS: Adversarial neural architecture search for GANs. arXiv preprint arXiv:1912.02037 (2019).

[4] Xinyu Gong, Shiyu Chang, Yifan Jiang, and Zhangyang Wang. 2019. AutoGAN: Neural architecture search for generative adversarial networks. In ICCV. 3224-3234.

[5] Martin Heusel, Hubert Ramsauer, Thomas Unterthiner, Bernhard Nessler, and Sepp Hochreiter. 2017. GANs trained by a two time-scale update rule converge to a local nash equilibrium. In NIPS. 6626-6637.

[6] Diederik P. Kingma and Jimmy Ba. 2015. Adam: A method for stochastic optimization. In ICLR

[7] Julian F. Miller and Stephen L. Smith. 2006. Redundancy and computational efficiency in cartesian genetic programming. IEEE Transactions on Evolutionary Computation 10, 2 (2006), 167-174

[8] Julian F. Miller and Peter Thomson. 2000. Cartesian genetic programming. In EuroGP. 121-132.

[9] Takeru Miyato, Toshiki Kataoka, Masanori Koyama, and Yuichi Yoshida. 2018. Spectral normalization for generative adversarial networks. In ICLR.

[10] Takeru Miyato and Masanori Koyama. 2018. cGANs with projection discriminator. In ICLR.

[11] Vinod Nair and Geoffrey E. Hinton. 2010. Rectified linear units improve restricted boltzmann machines. In ICML. 807-814.

[12] Andrew M. Saxe, James L. McClelland, and Surya Ganguli. 2013. Exact solutions to the nonlinear dynamics of learning in deep linear neural networks. In ICLR. 


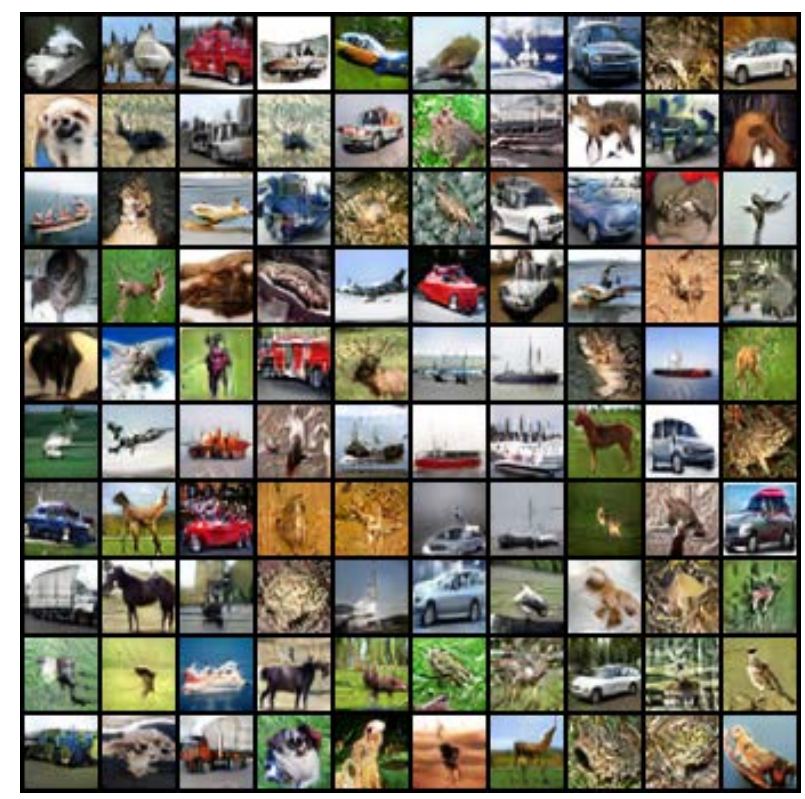

(a) Unsupervised image generation

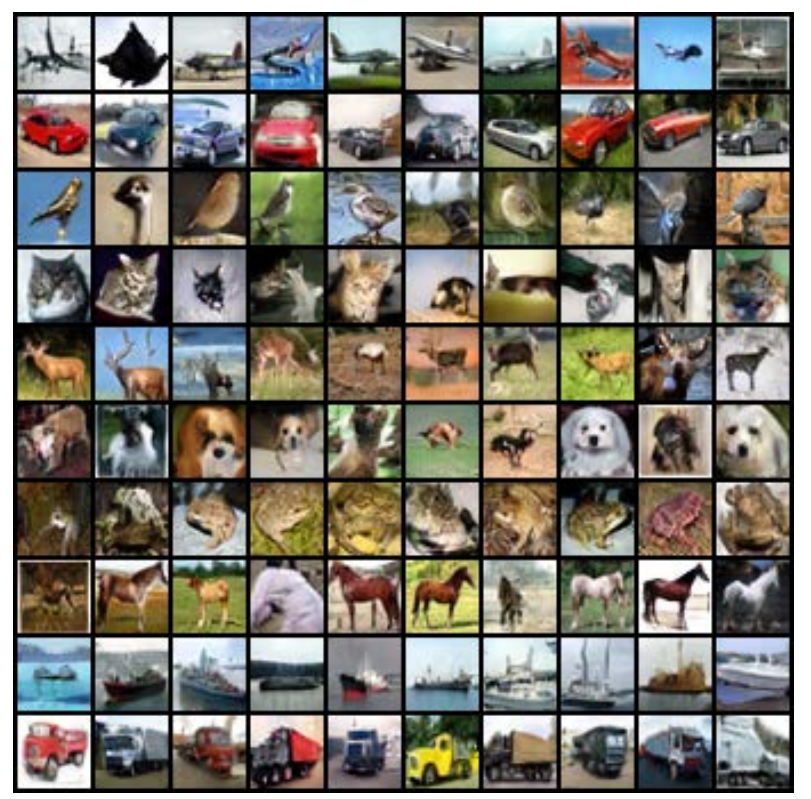

(b) Supervised image generation

Figure 2: Examples of CIFAR-10 images generated from our models.

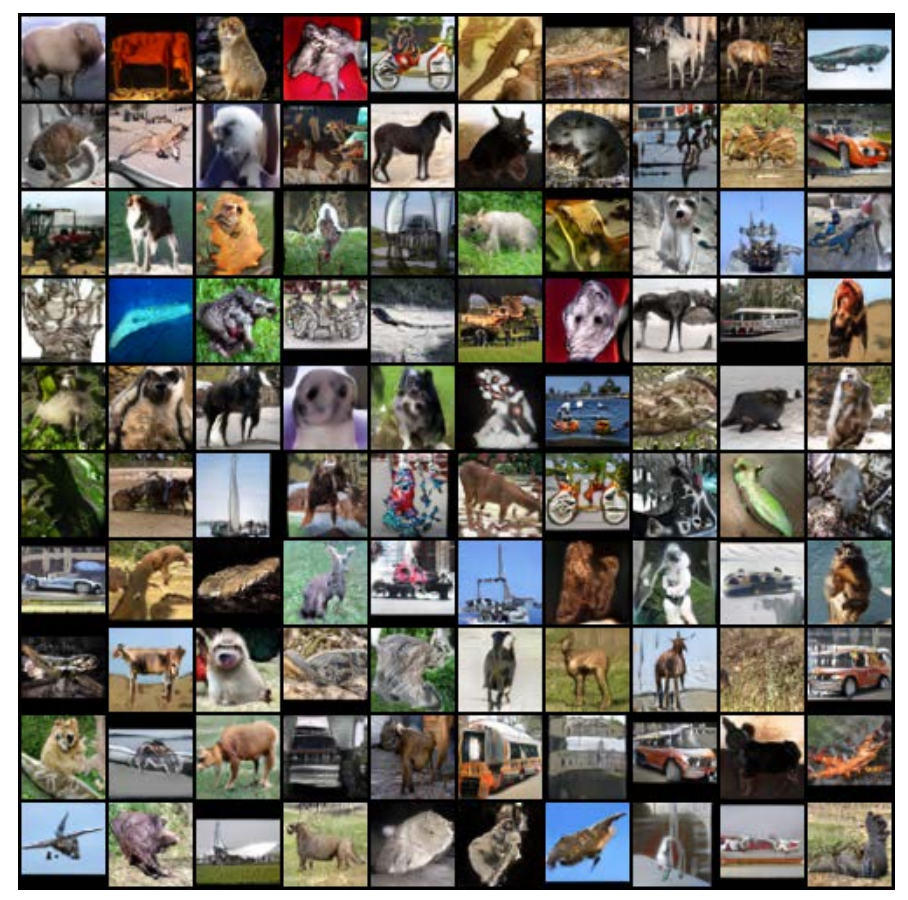

Figure 3: Examples of STL-10 images generated from our model with unsupervised setting. 